OPEN ACCESS

Edited by:

Desheng Liang,

Central South University, China

Reviewed by:

Yuan Shi,

Children's Hospital of Chongqing Medical University, China

Kejian Zhang

Mount Sinai Genomics, United States

${ }^{*}$ Correspondence:

Deyi Zhuang

zhuangdy526@163.com

Guang Lin

linguang_9999@126.com

Huijun Wang

huijunwang@fudan.edu.cn

tThese authors share first authorship

Specialty section:

This article was submitted to

Genetics of Common and Rare

Diseases,

a section of the journal

Frontiers in Pediatrics

Received: 18 June 2021 Accepted: 20 September 2021

Published: 18 October 2021

Citation:

Ma H, Tang Z, Xiao F, Li L, Li Y,

Tang W, Chen L, Kang W, Lu Y,

Dong $X$, Cheng G, Wang L, Lu W, Yang $L, N i$, Peng $X$, Wang $Y$, Cao $Y$, Wu B, Zhou W, Zhuang $D$, Lin $G$ and

Wang $H$ (2021) Neonatal Metabolic Acidosis in the Neonatal Intensive Care Unit: What Are the Genetic Causes? Front. Pediatr. 9:727301. doi: 10.3389/fped.2021.727301

\section{Neonatal Metabolic Acidosis in the Neonatal Intensive Care Unit: What Are the Genetic Causes?}

\author{
Haiyan $\mathrm{Ma}^{1+}$, Zezhong Tang ${ }^{2 \dagger}$, Feifan $\mathrm{XiaO}^{3}$, Long $\mathrm{Li}^{4}$, Yangfang $\mathrm{Li}^{5}$, Wenyan Tang ${ }^{6}$, \\ Liping Chen ${ }^{7}$, Wenqing Kang ${ }^{8}$, Yulan Lu $^{3}$, Xinran Dong ${ }^{3}$, Guoqiang Cheng ${ }^{9}$, \\ Laishuan Wang ${ }^{9}$, Wei Lu ${ }^{10}$, Lin Yang ${ }^{10}$, Qi Ni ${ }^{3}$, Xiaomin Peng ${ }^{3}$, Yao Wang ${ }^{3}$, Yun Cao ${ }^{9}$, \\ Bingbing $\mathrm{Wu}^{3}$, Wenhao Zhou ${ }^{3,9}$, Deyi Zhuang ${ }^{11 *}$, Guang Lin $^{1 *}$ and Huijun Wang ${ }^{3 *}$
}

\begin{abstract}
${ }^{1}$ Department of Neonatology, Zhuhai Women and Children's Hospital, Zhuhai, China, ${ }^{2}$ Department of Pediatrics, Peking University First Hospital, Beijing, China, ${ }^{3}$ Center for Molecular Medicine, Children's Hospital of Fudan University, Shanghai, China, ${ }^{4}$ Department of Neonatology, People's Hospital of Xinjiang Uygur Autonomous Region, Urumai, China, ${ }^{5}$ Department of Neonatology, Kunming Children's Hospital, Kunming, China, ${ }^{6}$ Department of Neonatology, Jiangxi Maternal Hospital, Nanchang, China, ${ }^{7}$ Department of Neonatology, Jiangxi Provincial Children's Hospital, Nanchang, China, ${ }^{8}$ Department of Neonatology, Children's Hospital Affiliated to Zhengzhou University, Zhengzhou, China, ${ }^{9}$ Department of Neonatology, Children's Hospital of Fudan University, Key Laboratory of Neonatal Diseases, Ministry of Health, Shanghai, China,

${ }^{10}$ Department of Endocrinology and Inherited Metabolic Diseases, Children's Hospital of Fudan University, Shanghai, China,

${ }^{11}$ Xiamen Key Laboratory of Neonatal Diseases, Xiamen Children's Hospital, Xiamen, China
\end{abstract}

Neonatal metabolic acidosis (NMA) is a common problem, particularly in critically ill patients in neonatal intensive care units (NICUs). Complex etiologies and atypical clinical signs make diagnosis difficult; thus, it is crucial to investigate the underlying causes of NMA rapidly and provide disorder-specific therapies. Our study aims to provide an overview of the genetic causes of NMA in patients from NICUs. We performed next-generation sequencing (NGS) on neonates with NMA from January 2016 to December 2019. Clinical features, genetic diagnoses, and their effects on clinical interventions were collected for analysis. In the 354 enrolled patients, 131 (37\%) received genetic diagnoses; 95 (72.5\%) of them were autosomal recessively inherited diseases. Two hundred and fifteen variants spanning 57 genes were classified as pathogenic (P) or likely pathogenic (LP) in 131 patients. The leading cause was metabolic disorders due to 35 genes found in 89 patients (68\%). The other 42 NMA patients (32\%) with 22 genes had malformations and renal, neuromuscular, and immune-hematological disorders. Seven genes (MMUT, MMACHC, CHD7, NPHS1, OTC, IVD, and PHOX2B) were noted in more than four patients, accounting for $48.9 \%$ (64/131) of the identified P/LP variants. Forty-six diagnosed patients with uncorrected NMA died or gave up. In conclusion, 37\% of neonates with metabolic acidosis had genetic disorders. Next-generation sequencing should be considered when investigating the etiology of NMA in NICUs. Based on early molecular diagnoses, valuable treatment options can be provided for some genetic diseases to achieve better outcomes.

Keywords: neonatal metabolic acidosis, neonatal intensive care units, next-generation sequencing, gene, neonate 


\section{INTRODUCTION}

Neonatal metabolic acidosis (NMA) is the accumulation of non-carbonic acid equivalents, which arises from excessive production or inadequate excretion of hydrogen ions or from an increased loss of bicarbonate (1). Neonatal metabolic acidosis is associated with poor clinical outcomes (2). The clinical features of NMA are atypical and are related to varying degrees of primary disease. The causes of NMA are complicated and varied, including birth asphyxia, cold stress, hypovolemia, sepsis, congenital heart disease, renal disease, and inborn errors of metabolism (1). Evaluation of detailed history, physical examination, and basic laboratory tests were insufficient to determine the cause. Administration of a fluid bolus or sodium bicarbonate is the initial management for correcting metabolic acidosis (3). These treatments do not deal with the cause but only correct the $\mathrm{pH}(3-6)$. Although interventions have been established in clinical practice, the impact on long-term neurological morbidity remains uncertain. With the increased awareness of the association between genotype and diseases, it has been found that infants with metabolic acidosis admitted to neonatal intensive care units (NICUs) are more likely to have genetic disorders. Thus, early genetic diagnosis is essential for neonates with NMA.

In recent years, next-generation sequencing (NGS) technology has been increasingly applied in clinical practice as a genetic diagnostic tool. Next-generation sequencing, especially exome sequencing and whole-genome sequencing, has shown advantages in facilitating an accurate diagnosis that is difficult to confirm using clinical or laboratory criteria for patients with disorders in the NICU $(7,8)$. Recent studies have reported enabling rapid sequencing at reduced costs and better performance in critically ill neonates $(9,10)$.

In this study, we analyzed the clinical medical records and genetic testing results of patients with NMA and summarized the clinical features, genetic diagnoses, and their effect on clinical intervention. Our study aims to provide a better understanding of the genetic causes of NMA that translate to more accurate management and better prognosis.

\section{MATERIALS AND METHODS \\ Study Design and Participants}

This was a retrospective cohort study conducted at the Clinical Genetics Laboratory of the Children's Hospital of Fudan University. Patients in NICUs were recruited retrospectively between January 1, 2016, and December 31, 2019. The inclusion criteria were as follows: (1) diagnosis of NMA based on arterial blood gas, $\mathrm{pH}<7.3, \mathrm{BE}<-10 \mathrm{mmol} / \mathrm{L}$, and $\mathrm{PaCO}_{2} 35-$ $45 \mathrm{mmHg}$; and (2) an order for NGS. Notably, patients with NMA who did not undergo NGS were excluded. In addition, patients were excluded if they were genetically diagnosed before enrollment. The clinical features of each patient were ascertained comprehensively by a physician in addition to a thorough review of their medical records. The clinical data included sex, major clinical features, and outcomes of metabolic acidosis. Pretest counseling was performed by physicians. This study was approved by the Medical Ethics Committee of the Children's Hospital of Fudan University (2015-130). Informed consent was obtained from the patients' parents or guardians.

\section{Next-Generation Sequencing}

Peripheral blood was collected, and genomic DNA was extracted using the TIANGEN DNA Blood Mini Kit according to the manufacturer's protocol. Sequences were generated using the Agilent ClearSeq Inherited Disease panel kit (including 2,742 genes) for clinical exome sequencing or the Agilent SureSelect XT Human All Exon V5 kit for exome sequencing. Nextgeneration sequencing was performed using the Illumina HiSeq X10 platform. The average on-target sequencing depth was $\times 200$ for the clinical exome sequencing and $\times 120$ for exome sequencing. Sequencing reads were mapped to the reference human genome (UCSC hg19) using the Burrows-Wheeler Aligner. A phenotype-scoring algorithm named PhenoPro was used for the variant filtering process (11). The detected variants were confirmed using PCR, and PCR-amplified DNA products were subjected to direct automated sequencing (3500XL Genetic Analyzer, Applied Biosystems) according to the manufacturer's specifications. De novo variants were detected by parental confirmation using Sanger sequencing. The pathogenicity of the variant was defined based on the American College of Medical Genetics and Genomics criteria (12). Detailed methods can be found in our previous studies $(13,14)$.

\section{Statistical Analysis}

Frequency count and proportions were used for categorical data. Data were analyzed using Pearson's $\chi^{2}$ independence test. Statistical significance was set at a $p$-value $<0.05$. All statistical analyses were conducted using IBM SPSS version 20 (IBM Corp., Armonk, NY, USA).

\section{RESULTS}

\section{Demographics of Clinical Features}

From January 1, 2016, to December 31, 2019, 733 patients were clinically diagnosed with metabolic acidosis in NICUs. Three cases had genetic diagnoses, 38 neonates did not undergo NGS because their parents declined, and 338 patients did not undergo NGS for other reasons. Finally, 354 (48.3\%) neonates with NGS were enrolled and classified as diagnosed and undiagnosed according to the genetic findings of our analysis (Figure 1). Of the enrolled patients, 186 (52.5\%) were males and $168(47.5 \%)$ were females. The major clinical manifestations (one patient may have more than one clinical feature) were neuromuscular dysfunction (49.2\%), cardiopulmonary dysfunction (37.3\%), hepatorenal disorder (17.2\%), infection (13.6\%), and malformation (12.1\%) (Table 1).

\section{Genetic Diagnosis}

Of these 354 neonates, 131 (37\%) received genetic diagnoses through NGS, while the remaining did not $(n=223,63 \%)$. The malformation rate was higher in the diagnosed group $(9.1 \%$, $p=0.002)$ than in the undiagnosed group $(8.0 \%)$. However, 


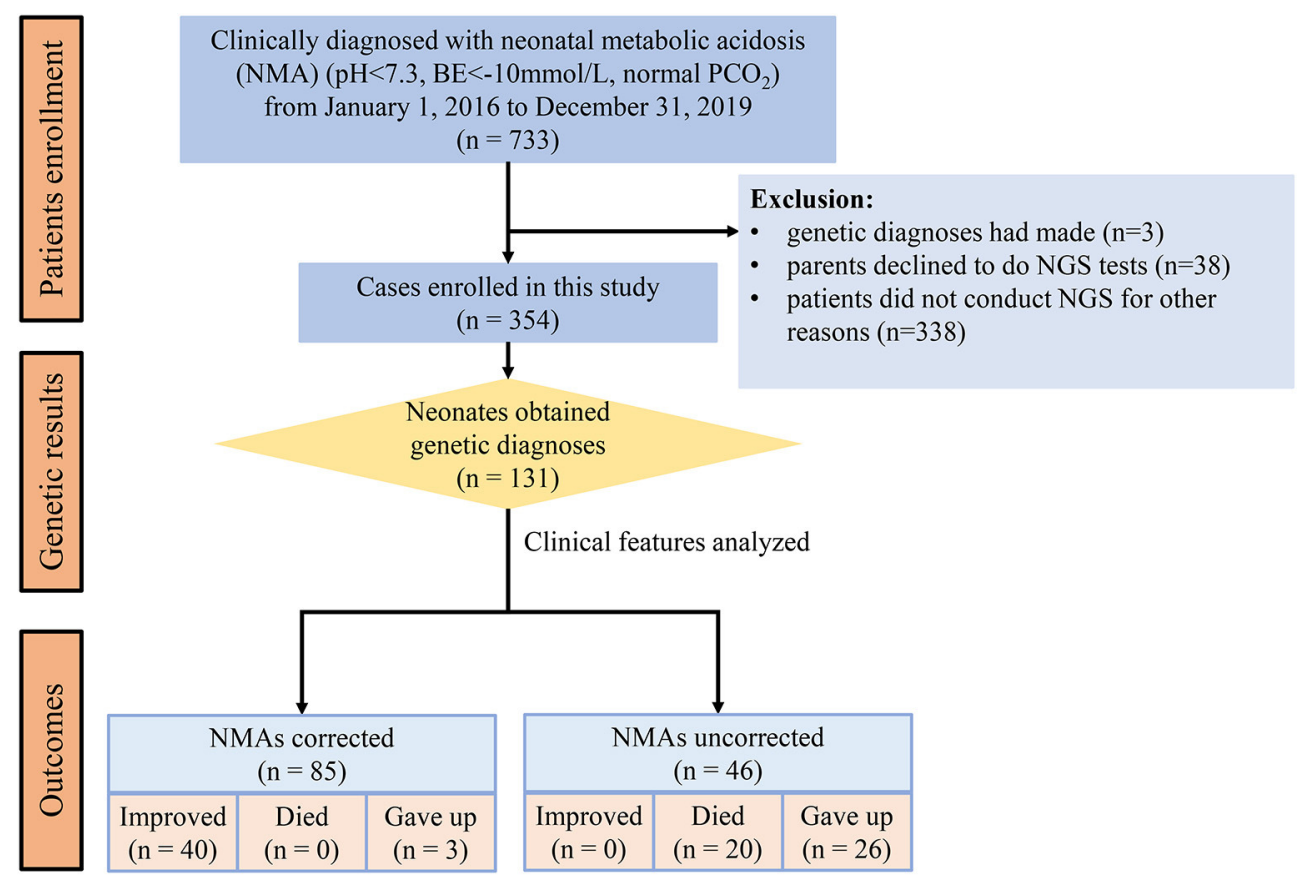

FIGURE 1 | Flowchart of diagnosed patients by NGS.

TABLE 1 | Demographic features and phenotypes of NMA patients tested with NGS.

\begin{tabular}{|c|c|c|c|c|}
\hline Characteristics & $\begin{array}{c}\text { Total } \\
N(\%) \\
(\text { Total }=354)\end{array}$ & $\begin{array}{c}\text { Diagnosed } \\
N(\%) \\
\text { (Total = 131) }\end{array}$ & $\begin{array}{c}\text { Undiagnosed } \\
\qquad N(\%) \\
(\text { Total }=223)\end{array}$ & $p$-value \\
\hline Sex & & & & 0.043 \\
\hline Male & $186(52.5)$ & $78(59.5)$ & $108(48.4)$ & \\
\hline Female & $168(47.5)$ & $53(40.5)$ & $115(51.6)$ & \\
\hline \multicolumn{5}{|l|}{$\begin{array}{l}\text { Major clinical features by } \\
\text { systems* }\end{array}$} \\
\hline $\begin{array}{l}\text { Neuromuscular } \\
\text { dysfunction }\end{array}$ & $174(49.2)$ & $56(42.7)$ & $118(52.9)$ & 0.065 \\
\hline $\begin{array}{l}\text { Cardiorespiratory } \\
\text { dysfunction }\end{array}$ & $132(37.3)$ & $45(34.3)$ & $87(39.0)$ & 0.381 \\
\hline Hepatorenal disorder & $61(17.2)$ & $22(16.8)$ & $39(17.5)$ & 0.867 \\
\hline Infection & 48 (13.6) & $20(15.3)$ & $28(12.6)$ & 0.472 \\
\hline Malformation & $43(12.1)$ & $25(19.1)$ & $18(8.1)$ & 0.002 \\
\hline $\begin{array}{l}\text { Outcomes of metabolic } \\
\text { acidosis }\end{array}$ & & & & 0.63 \\
\hline Been corrected & 224 (63.3) & $85(64.9)$ & 139 (62.3) & \\
\hline Uncorrected & $130(36.7)$ & $46(35.1)$ & $84(37.7)$ & \\
\hline \multicolumn{5}{|l|}{ Overall outcomes } \\
\hline Improved & $149(42.1)$ & $40(30.5)$ & 109 (48.9) & 0.001 \\
\hline $\begin{array}{l}\text { Gave up medical } \\
\text { support }\end{array}$ & $55(15.5)$ & $29(22.1)$ & $26(11.7)$ & 0.009 \\
\hline Died with diseases & $52(14.7)$ & $20(15.3)$ & 32 (14.3) & 0.814 \\
\hline
\end{tabular}

*One patient may have more than one clinical feature. $p$-values are obtained from a $\chi^{2}$-test. there were no other significantly different major clinical features between the diagnosed and undiagnosed groups (Table 1).

In 131 patients with genetic diagnoses, 215 variants spanning 57 genes were classified as pathogenic (P) or likely pathogenic (LP). These patients were diagnosed with a monogenetic disorder, with 95 autosomal recessive cases, which included 80 compound heterozygous and 15 homozygous cases, 22 autosomal dominants with eight cases identified as de novo, $12 \mathrm{X}$-link recessive cases with hemizygote, and two $\mathrm{X}$-link dominants with heterozygous cases.

According to the 57 phenotype-genotype-related diagnoses, diseases were classified into six categories: metabolic, renal, neuromuscular, and immune-hematological disorders; malformation; and others. Metabolic disorder was the most common disease found in $89(67.9 \%)$ patients, followed by malformation in $22(16.8 \%)$ patients. Renal, neuromuscular, and immune-hematological disorders were detected in nine (6.9\%), six (4.6\%), and three (2.3\%) patients, respectively. Two other genes were detected in two patients (Figure 2). Seven genes with $\mathrm{P} / \mathrm{LP}$ variants identified in more than four patients were MMUT, MMACHC, CHD7, NPHS1, OTC, IVD, and PHOX2B, accounting for $48.9 \%(64 / 131)$ of the patients in our cohort (Figure 3).

Of the metabolic disorders, 35 genes were classified into eight subgroups: amino acids, organic acids, sugar, fatty acids, carnitine, urea cycle, vitamin, and mitochondria disorders. Eleven genes were responsible for organic acid disorders, covering 53.9\% (48/89) of the metabolic disorder cases. Four genes (OTC, ASL, CPS1, and ASS1) were responsible for urea 


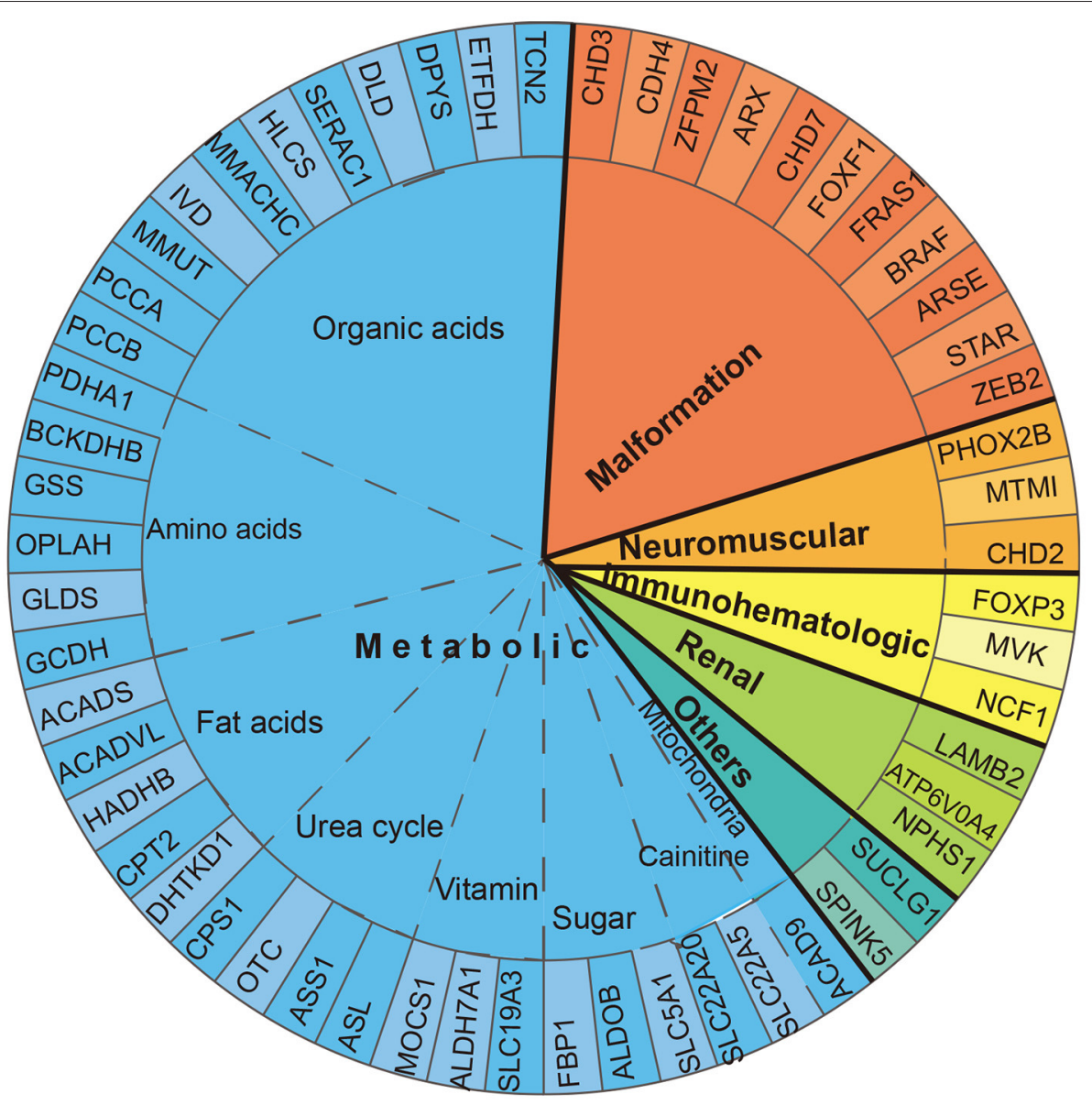

FIGURE 2 | Diagnosed gene distribution according to the disease. Fifty-seven genes are displayed by classification. Thirty-five genes related to metabolic disorder were classified into eight subgroups: organic acid, amino acid, fat acid, urea cycle, sugar, vitamin, carnitine, and mitochondria disorder.

cycle disorders, accounting for 14.6\% (13/89). Fatty acid, amino acid, and carnitine disorders were detected in eight (9\%), eight (9\%), and five (5.6\%) patients, respectively. Sugar and vitamin disorders were found in three $(3.3 \%)$ patients. Mitochondria disorders were found in a patient (1.1\%) (Figure 2).

\section{Clinical Intervention and Outcomes}

Metabolic acidosis was clinically corrected in 224 (63.3\%) patients. However, metabolic acidosis was not corrected in $130(36.7 \%)$ patients, including 46 diagnosed and 84 undiagnosed neonates. In the improved outcome cases, the rate of improvement was higher in the undiagnosed group (48.9\%) than in the diagnosed group $(30.5 \%)(p<0.01)$. In contrast, the rate of patients who gave up medical support was higher in the diagnosed group ( 22.1 vs. $11.6 \%, p<0.01$ ) (Table 1 ). In the 131 diagnosed cases, 46 neonates with uncorrected NMAs all died or gave up. However, only three cases gave up, and no case died in the 85 neonates with corrected NMAs (Figure 1).

Of the 52 deaths in this study, 38.5\% (20/52) received a genetic diagnosis (Table 1). Of these, 5 (25\%) were diagnosed postmortem because their symptoms developed and progressed rapidly, resulting in mortality $24 \mathrm{~h}$ after admission. Through genetic diagnosis, accurate diagnoses can explain the deaths. OTC was the cause of death due to hyperammonemia in case NMA011 and encephalopathy and sepsis in case NMA047. Case NMA069, who carried two missense variants in $A C A D V L$, died from cardiopulmonary arrest. Case NMA002 with encephalopathy and multiple organ failure was identified as a missense variant of GSS.

In addition, 40 patients with genetic diagnoses may receive precise treatment options. Similar to case NMA074, a 28-dayold male patient presented with poor feeding and lethargy. $\mathrm{He}$ was diagnosed with distal renal tubular acidosis after genetic identification with compound heterozygous variants in the ATP6V0A4 gene. If the baby was not treated accurately and was followed up regularly, he would have developed severe complications, which would affect growth and development. Case NMA036, who was a 27-day-old male infant with lethargy and poor weight gain, was admitted to NICU. Blood tandem mass spectrometry showed increased tyrosine level, and the rest of the routine tests were highly suspicious for infection. Finally, methylmalonic acidemia (MMA) and homocystinuria, cblC type, were diagnosed based on genetic findings. The infant had improved upon targeted management. NMA003 was an 8day-old baby boy who presented with vomiting, hypoglycemia, 


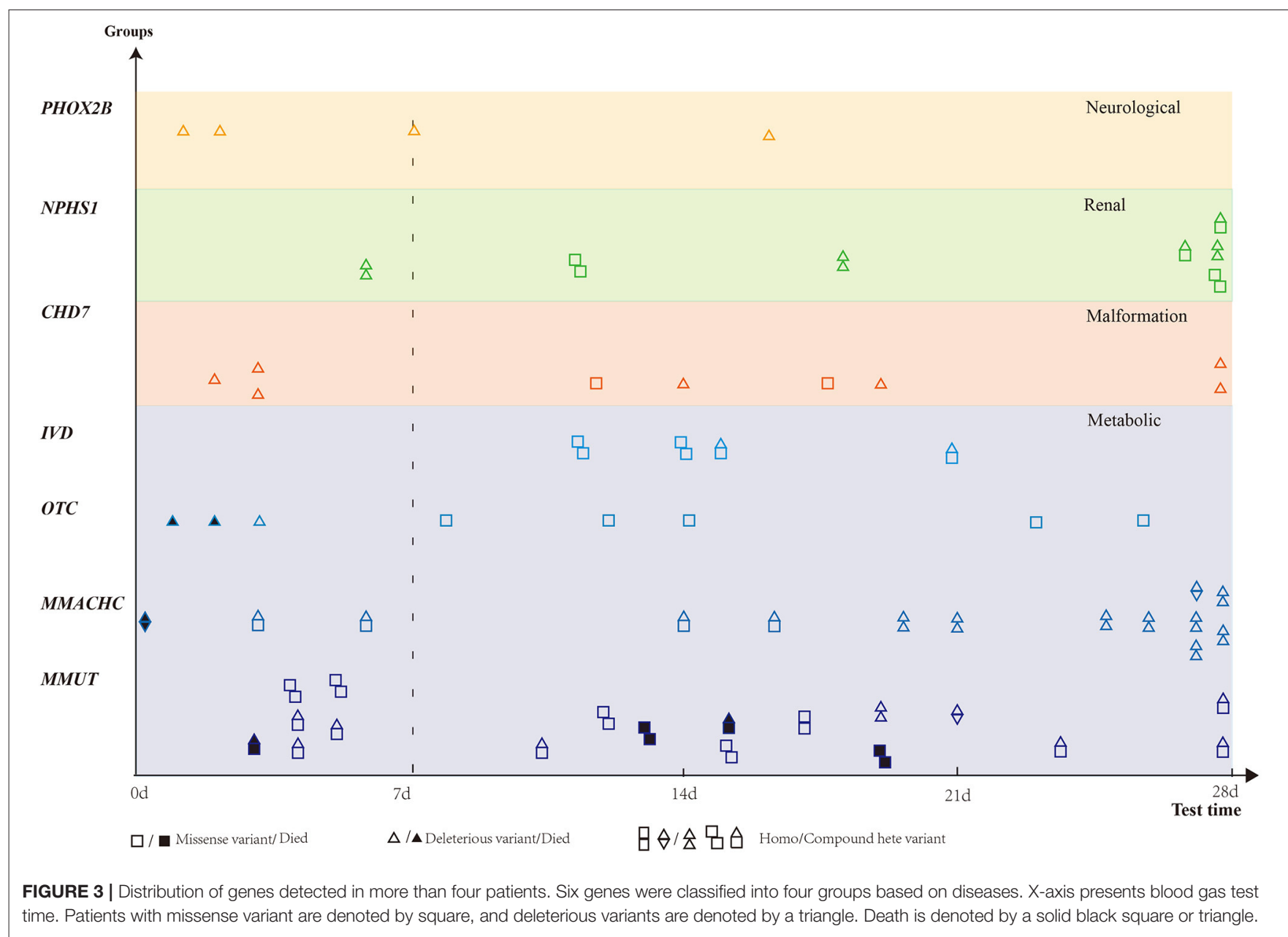

and seizures. A high level of tyrosine was found in blood mass spectrometry, and tyrosine metabolism disorder was more likely to be diagnosed. However, a hemizygote variation (G71E) was detected in the OTC gene by NGS and was classified as LP. The baby was diagnosed with ornithine transcarbamylase deficiency (OTCD, MIM 311250) based on genetic findings. The patient survived on a new effective treatment option. Case NMA111, who was a 20-day-old female infant with hepatic dysfunction and suspected sepsis, had compound heterozygous variants in the gene $A L D O B$. The patient was diagnosed with hereditary fructose intolerance (HFI, MIM 229600). The disease generally develops after feeding in the neonatal period. Severity is related to the amount and duration of fructose consumption. The baby was treated immediately with a fructose intake-controlled diet, which can prevent life-threatening complications and avoid invasive procedures such as liver biopsy. The baby can grow into adulthood on a well-controlled diet.

\section{DISCUSSION}

In our study, 131 of 354 (37\%) neonates with metabolic acidosis received a genetic diagnosis. In total, 57 genes were responsible for a wide range of diseases, from seemingly healthy infants to critically ill patients. Different degrees of primary disease lead to varied and atypical clinical presentations. Complex etiologies and non-specific clinical signs made diagnosis more difficult.

The leading cause of NMA was metabolic disorders, which involved 35 genes, covering $68 \%$ of genetically diagnosed patients. Only $38(38 / 89,42.9 \%)$ patients had clinical diagnoses before receiving genetic diagnoses. Organic acid disorder is the major cause of metabolic acidosis. The top two genes, MMUT and $M M A C H C$, resulted in MMA that was related to organic acid disorder. A total of 24 (24/32, 75\%) MMA patients were diagnosed with MMA using mass spectrometric tests before NGS. Next-generation sequencing can help confirm diagnoses and determine the subtype of organic acid disorder. Phenotypegenotype correlations may predict disease severity depending on mRNA stability and protein residual function (15). Variations in MMUT were identified as MMUT(0) phenotypes, indicating no detectable enzymatic activity (16). Variations in MMACHC have been detected as MMA and homocystinuria, cblC type (15). Patients who are compound heterozygotes for a missense allele appear to have a milder phenotype (15). Variation analysis was associated with responses to vitamin B12. Generally, the cblC type is almost entirely vitamin $\mathrm{B} 12$ responsive, the $\operatorname{MMUT}(0)$ type is vitamin B12 unresponsive, and other types are partly 
responsive to vitamin $\mathrm{B} 12$ (17). IVD variations were found in four patients; three of them were diagnosed with isovaleric acidemia by mass spectrometric tests before NGS. Genetic findings confirmed the diagnoses and treatment. OTC variation results in OTCD, which accounts for approximately half of the urea cycle defects (18). As our data presented, OTC variations were detected in eight patients. Three of these patients were diagnosed with urea cycle defects by mass spectrometric tests before NGS. Genetic findings may help physicians understand the progress of OTCD. Six patients with missense variants that can reduce OTC enzymatic activity or stability survived OTCD (18). Patient NMA047 died $24 \mathrm{~h}$ after admission. He was found to carry a splicing variant, affecting mRNA processing and decreasing OTC enzyme levels (18).

Interestingly, in 42 diagnosed patients (42/131, 32\%), NMA was caused by malformation and renal, neuromuscular, and immune-hematological disorders, and not by metabolic disorders. P/LP variations in NPHS1, which encodes nephrin, were detected in seven patients with massive proteinuria, edema, infection, and poor feeding or respiratory distress. NPHS1 is the main underlying cause of congenital nephritic syndrome, which is associated with high morbidity and mortality (19). Genetic diagnosis provides precise information on treatment and benefits patient outcomes. Pathogenic variants of $\mathrm{CHD} 7$ were detected in nine neonates with infection, feeding difficulty, respiratory distress, and one or more malformations, which led to severe metabolic acidosis. These patients should be treated as if they are diagnosed with CHARGE syndrome (20). However, physicians will not give the diagnosis of CHARGE syndrome without genetic basis because criteria that focus on typical clinical phenotypes may exclude patients with a mild phenotype in the neonatal period. It has been proposed that pathogenic $\mathrm{CHD} 7$ variant status is now a major criterion in CHARGE syndrome diagnoses (21). In four patients with recurrent apnea, infection, hypoxemia, and pathogenic variants, $P H O X 2 B$ was identified. Genetic tests help physicians and families find the cause of diseases and help predict progress thereafter (22). Our study showed that mass spectrometric tests were insufficient for investigating the etiology of NMA, as opposed to NGS.

Based on early molecular diagnoses, valuable treatment options can be provided for some genetic diseases, and patients can survive or achieve better outcomes. For case NMA074, genetic diagnosis (distal renal tubular acidosis) was performed before the appearance of severe complications. With earlier adequate metabolic control, the infant can experience better growth and kidney function (23). Case NMA003, an 8-day-old male infant with abnormal mass spectrometric tests, was highly suspected to have metabolic disorder. OTCD was confirmed by genetic testing and appropriately treated. Case NMA036, a 27-day-old infant, underwent many blood tests and invasive procedures. The infant did not improve on anti-infection treatment but did improve with targeted treatment of MMA, which was diagnosed by NGS. For case NMA111, the female infant only developed clinical symptoms when she was exposed to fructose as a monosaccharide, sucrose, or sorbitol (24). The patient recovered after a fructose-controlled diet, and liver biopsy was no longer necessary. These are the most desirable results for physicians and families and help push research into new therapies.

Although there was no difference between diagnosed and undiagnosed patients, if we compare the outcomes of NMA, the genetic test can effectively identify the etiology of the disease, and these precise diagnoses enable physicians and families to understand the cause of disease and the unavoidable poor clinical outcomes (25). Genetic counseling for families provided detailed progress, helped parents identify family carriers, and facilitated better planning of reproductive choices based on the risk of familial recurrence (26). The families in our study may benefit from previous genetic diagnoses and genetic counseling.

Based on our analysis, early genetic diagnosis is essential for neonates with NMA. We proposed that NGS be performed as early as possible when patients suffer from uncorrected metabolic acidosis and combine with one of the following items: (1) abnormal blood mass spectrometry or other abnormal biochemical markers such as blood ammonia or blood lactic acid; and (2) NMA neonates accompanied with malformation. For other NMA neonates, we suggest that NGS tests such as clinical exome sequencing should be ordered when physicians think that the NMA is a genetic factor involved.

This study had two limitations that should be addressed. First, although we designed to test all NMA patients in the NICU, we had 338 cases that did not undergo NGS for different reasons. Second, as a multicenter study, follow-up information was limited, and the outcomes of some patients were missed.

\section{CONCLUSION}

In our study, we found that metabolic acidosis in approximately $37 \%$ of neonates in NICU was caused by genetic disorders. Nextgeneration sequencing should be considered when investigating the etiology of NMA. Based on early molecular diagnoses, valuable treatment options can be provided for some genetic diseases to achieve better outcomes.

\section{ETHICS STATEMENT}

The studies involving human participants were reviewed and approved by Medical Ethics Committee of Children's Hospital of Fudan University (2015-130). Written informed consent to participate in this study was provided by the participants' legal guardian/next of kin. Written informed consent was obtained from the minor(s)' legal guardian/next of kin for the publication of any potentially identifiable images or data included in this article.

\section{AUTHOR CONTRIBUTIONS}

HW, DZ, and GL designed and supervised the overall study. FX, YLu, XD, GC, LW, WL, LY, QN, XP, and YW conducted analysis on the aggregated large cohort data. LL, YLi, WT, LC, WK, YC, BW, and WZ collected, 
supervised, and reviewed the clinical data. HM and ZT wrote the original manuscript draft. HW critically reviewed and revised the manuscript for important intellectual

\section{REFERENCES}

1. Walter JH. Metabolic acidosis in newborn infants. Arch Dis Child. (1992) 67(7 Spec No):767-9. doi: 10.1136/adc.67.7_spec_no.767

2. Kraut JA, Madias NE. Treatment of acute metabolic acidosis: a pathophysiologic approach. Nat Rev Nephrol. (2012) 8:589-601. doi: $10.1038 /$ nrneph.2012.186

3. Lawn CJ, Weir FJ, McGuire W. Base administration or fluid bolus for preventing morbidity and mortality in preterm infants with metabolic acidosis. Cochrane Database Syst Rev. (2005) (2):CD003215. doi: 10.1002/14651858.CD003215.pub2

4. Aschner JL, Poland RL. Sodium bicarbonate: basically useless therapy. Pediatrics. (2008) 122:831-5. doi: 10.1542/peds.2007-2400

5. Rochwalsky U, Seitz C, Heinzmann T, Poeschl J, Koch L. [Correction of acidosis in neonatal intensive-care medicine: a national survey]. Klin Padiatr. (2015) 227:219-24. doi: 10.1055/s-0034-1396866

6. Collins A, Sahni R. Uses and misuses of sodium bicarbonate in the neonatal intensive care unit. Semin Fetal Neonat Med. (2017) 22:336-41. doi: 10.1016/j.siny.2017.07.010

7. Meng L, Pammi M, Saronwala A, Magoulas P, Ghazi AR, Vetrini F, et al. Use of exome sequencing for infants in intensive care units: ascertainment of severe single-gene disorders and effect on medical management. JAMA Pediatr. (2017) 171:e173438. doi: 10.1001/jamapediatrics.2017.3438

8. Smith HS, Swint JM, Lalani SR, Yamal JM, de Oliveira Otto MC, Castellanos $\mathrm{S}$, et al. Clinical application of genome and exome sequencing as a diagnostic tool for pediatric patients: a scoping review of the literature. Genet Med. (2019) 21:3-16. doi: 10.1038/s41436-018-0024-6

9. Elliott AM, du Souich C, Lehman A, Guella I, Evans DM, Candido T, et al. RAPIDOMICS: rapid genome-wide sequencing in a neonatal intensive care unit-successes and challenges. Eur J Pediatr. (2019) 178:1207-18. doi: 10.1007/s00431-019-03399-4

10. Wang H, Lu Y, Dong X, Lu G, Cheng G, Qian Y, et al. Optimized trio genome sequencing (OTGS) as a first-tier genetic test in critically ill infants: practice in China. Hum Genet. (2020) 139:473-82. doi: 10.1007/s00439-019-02103-8

11. Li Z, Zhang F, Wang Y, Qiu Y, Wu Y, Lu Y, et al. PhenoPro: a novel toolkit for assisting in the diagnosis of Mendelian disease. Bioinformatics. (2019) 35:3559-66. doi: 10.1093/bioinformatics/btz100

12. Richards S, Aziz N, Bale S, Bick D, Das S, Gastier-Foster J, et al. Standards and guidelines for the interpretation of sequence variants: a joint consensus recommendation of the American College of Medical Genetics and Genomics and the Association for Molecular Pathology. Genet Med. (2015) 17:405-24. doi: 10.1038/gim.2015.30

13. Yang L, Kong $\mathrm{Y}$, Dong $\mathrm{X}$, Hu L, Lin $\mathrm{Y}$, Chen $\mathrm{X}$, et al. Clinical and genetic spectrum of a large cohort of children with epilepsy in China. Genet Med. (2019) 21:564-71. doi: 10.1038/s41436-018-0 091-8

14. Wang H, Xiao F, Dong X, Lu Y, Cheng G, Wang L, et al. Diagnostic and clinical utility of next-generation sequencing in children born with multiple congenital anomalies in the China neonatal genomes project. Hum Mutat. (2021) 42:434-44. doi: 10.1002/humu.24170

15. Carrillo-Carrasco N, Chandler RJ, Venditti CP. Combined methylmalonic acidemia and homocystinuria, cblC type. I. Clinical presentations, diagnosis and management. J Inherit Metab Dis. (2012) 35:91-102. doi: 10.1007/s10545-011-9364-y content. All authors approved the final manuscript as submitted and agreed to be accountable for all aspects of the work.
16. Almási T, Guey LT, Lukacs C, Csetneki K, Vokó Z, Zelei T. Systematic literature review and meta-analysis on the epidemiology of methylmalonic acidemia (MMA) with a focus on MMA caused by methylmalonylCoA mutase (mut) deficiency. Orphan J Rare Dis. (2019) 14:84. doi: 10.1186/s13023-019-1063-Z

17. Zhou X, Cui Y, Han J. Methylmalonic acidemia: current status and research priorities. Intract Rare Dis Res. (2018) 7:73-8. doi: 10.5582/irdr.2018.01026

18. Caldovic L, Abdikarim I, Narain S, Tuchman M, Morizono H. Genotypephenotype correlations in ornithine transcarbamylase deficiency: a mutation update. J Genet Genomics. (2015) 42:181-94. doi: 10.1016/j.jgg.2015.04.003

19. Sharief SN, Hefni NA, Alzahrani WA, Nazer II, Bayazeed MA, Alhasan $\mathrm{KA}$, et al. Genetics of congenital and infantile nephrotic syndrome. World J Pediatr. (2019) 15:198-203. doi: 10.1007/s12519-018-00224-0

20. Hsu P, Ma A, Wilson M, Williams G, Curotta J, Munns CF, et al. CHARGE syndrome: a review. J Paediatr Child Health. (2014) 50:504-11. doi: $10.1111 /$ jpc. 12497

21. Hale CL, Niederriter AN, Green GE, Martin DM. Atypical phenotypes associated with pathogenic CHD7 variants and a proposal for broadening CHARGE syndrome clinical diagnostic criteria. Amer J Med Genet A. (2016) 170A:344-54. doi: 10.1002/ajmg.a.37435

22. Bishara J, Keens TG, Perez IA. The genetics of congenital central hypoventilation syndrome: clinical implications. Appl Clin Genet. (2018) 11:135-44. doi: 10.2147/TACG.S140629

23. Lopez-Garcia SC, Emma F, Walsh SB, Fila M, Hooman N, Zaniew M, et al. Treatment and long-term outcome in primary distal renal tubular acidosis. Nephrol Dial Transplant. (2019) 34:981-91. doi: 10.1093/ndt/gfy409

24. Tran C. Inborn errors of fructose metabolism. What can we learn from them? Nutrients. (2017). 9:356. doi: 10.3390/nu9040356

25. Bowdin SC. The clinical utility of next-generation sequencing in the neonatal intensive care unit. CMAJ. (2016) 188:786-7. doi: 10.1503/cmaj.160490

26. Borghesi A, Mencarelli MA, Memo L, Ferrero GB, Bartuli A, Genuardi $\mathrm{M}$, et al. Intersociety policy statement on the use of whole-exome sequencing in the critically ill newborn infant. Ital J Pediatr. (2017) 43:100. doi: 10.1186/s13052-017-0418-0

Conflict of Interest: The authors declare that the research was conducted in the absence of any commercial or financial relationships that could be construed as a potential conflict of interest.

Publisher's Note: All claims expressed in this article are solely those of the authors and do not necessarily represent those of their affiliated organizations, or those of the publisher, the editors and the reviewers. Any product that may be evaluated in this article, or claim that may be made by its manufacturer, is not guaranteed or endorsed by the publisher.

Copyright (C) 2021 Ma, Tang, Xiao, Li, Li, Tang, Chen, Kang, Lu, Dong, Cheng, Wang, Lu, Yang, Ni, Peng, Wang, Cao, Wu, Zhou, Zhuang, Lin and Wang. This is an open-access article distributed under the terms of the Creative Commons Attribution License (CC BY). The use, distribution or reproduction in other forums is permitted, provided the original author(s) and the copyright owner(s) are credited and that the original publication in this journal is cited, in accordance with accepted academic practice. No use, distribution or reproduction is permitted which does not comply with these terms. 